\title{
The Role of the Amygdala-Striatal Pathway in the Acquisition and Performance of Goal-Directed Instrumental Actions
}

\author{
Laura H. Corbit, ${ }^{1}$ Beatrice K. Leung, ${ }^{2}$ and Bernard W. Balleine ${ }^{2}$ \\ ${ }^{1}$ School of Psychology, and ${ }^{2}$ Brain and Mind Research Institute, The University of Sydney, Sydney NSW 2006, Australia
}

The posterior dorsomedial striatum (pDMS) is essential for the acquisition and expression of the specific response- outcome (R-0) associations that underlie goal-directed action. Here we examined the role of a pathway linking the basolateral amygdala (BLA) and pDMS in such goal-directed learning. In Experiment 1, rats received unilateral lesions of the BLA and were implanted with cannula targeting the pDMS in either the ipsilateral (control) or contralateral (disconnection) hemisphere. After initial training, rats received infusions of muscimol to inactivate the pDMS immediately before sessions in which novel R-0 associations were introduced. Sensitivity to devaluation by specific satiety was then assessed. Whereas rats in the ipsilateral group used the recently acquired associations to direct performance following devaluation, those in the contralateral group could not, indicating that BLA-pDMS disconnection prevented the acquisition of the new R-0 associations. Indeed, evidence suggested that these rats relied instead on learning acquired during prior training to direct performance following devaluation. In Experiment 2, rats underwent similar surgery and training except they received muscimol infusions immediately before devaluation testing. Those in the ipsilateral group showed a selective devaluation effect, again based on the most recently introduced R-0 associations. In contrast, rats in the contralateral group showed nonselective performance after devaluation indicating that the BLA-DMS pathway is also required for the expression of selective R-0 associations. Together these results suggest that input from the BLA is essential for specific R-O learning by the pDMS.

\section{Introduction}

Previous work has shown that the medial region of the dorsal striatum (DMS) is critical for the acquisition and performance of goal-directed actions (Yin et al., 2005; Corbit and Janak, 2010; Shiflett and Balleine, 2010). For example, if the DMS is inactivated before sessions in which novel response-outcome ( $\mathrm{R}-\mathrm{O})$ associations are introduced, rats fail to encode these associations and fail to show selective responding when the value of one of those outcomes is subsequently reduced by devaluation (Corbit and Janak, 2010). In contrast, inactivation of the DLS is without effect, demonstrating that inactivation of DMS is responsible for this learning.

These regional differences likely result from different anatomical connections of dorsal striatal subregions (Alexander et al., 1986; Parent and Hazrati, 1995; Voorn et al., 2004; Haber et al., 2006). Of particular interest are inputs from the basolateral complex of the amygdala (BLA). The BLA has been reported to project broadly throughout the striatum; however, the density of the

\footnotetext{
Received Aug. 1, 2013; revised Sept. 25, 2013; accepted Sept. 29, 2013.

Author contributions: L.H.C. and B.W.B. designed research; L.H.C. and B.K.L. performed research; L.H.C. and B.K.L. analyzed data; L.H.C., B.K.L., and B.W.B. wrote the paper.

The research reported in this paper was supported by a grant from the Australian National Health and Medical Research Council NHMRC no. 633267).

The authors declare no completing financial interests.

Correspondence should be addressed to Dr Laura Corbit, School of Psychology, Brennan MacCallum Building (A18), University of Sydney NSW 2006, Australia. E-mail: laura.corbit@sydney.edu.au.

DOI:10.1523/JNEUROSCI.3271-13.2013

Copyright $\odot 2013$ the authors $\quad 0270-6474 / 13 / 3317682-09 \$ 15.00 / 0$
}

BLA inputs differs greatly depending on the striatal subregion (Kelley et al., 1982). Notably, the most anterior and lateral region of the DS (including the DLS region targeted in related behavioral studies) does not receive particularly dense BLA inputs, which are markedly more robust in the medial and posterior DMS (i.e., the pDMS).

Inputs from the BLA are of interest because of data suggesting that the BLA is responsible for assigning motivational significance to stimuli and responses, particularly when animals must discriminate multiple rewards with reference to their sensoryspecific properties (Balleine et al., 2003; Corbit and Balleine, 2005; Balleine and Killcross, 2006; Johnson et al., 2009). Whereas rats with BLA lesions are not impaired in the acquisition of instrumental tasks, they do show deficits in performance following outcome devaluation (Balleine et al., 2003; Corbit and Balleine, 2005). Information about the unique sensory and rewarding properties of different outcomes should be necessary for the learning performed by the pDMS; thus, we hypothesized that input from the BLA to the pDMS may be required for formation of specific $\mathrm{R}-\mathrm{O}$ relationships and for the control of selective responding.

The aim of the following experiments was explicitly to test the role of the connection between the BLA and DMS in the acquisition and expression of selective $\mathrm{R}-\mathrm{O}$ associations. We first confirmed the pathway between the BLA and the pDMS using retrograde tracing and c-Fos immunohistochemistry assessed after outcome devaluation testing. We next used a "disconnection" procedure in which we performed excitotoxic lesions of the BLA 
in one hemisphere and, before critical training or test sessions, inactivated the pDMS in the opposite hemisphere so as to functionally disrupt the BLA-pDMS pathway in both hemispheres. If connectivity between the BLA and pDMS is essential for R-O learning, we predicted that disruption of this pathway before training should prevent new R-O learning and consequently impair performance following devaluation. Similarly, if this pathway is essential for the expression of $\mathrm{R}-\mathrm{O}$ associations, disruption of this pathway before testing should also impair performance.

\section{Materials and Methods \\ Experiment 1: the role of the BLA-pDMS pathway in the acquisition of $\mathrm{R}-\mathrm{O}$ associations}

Experiment 1 aimed to confirm prior reports and our hypothesized function of the pathway linking the BLA and the pDMS in the acquisition of specific $\mathrm{R}-\mathrm{O}$ associations. Because previous tracing studies have suggested quite varied density of connections between the BLA and specific regions of the striatum, we thought it important to verify that a reasonably robust projection between the BLA and the specific region of the pDMS, that has previously been demonstrated to be critical for $\mathrm{R}-\mathrm{O}$ learning, exists and to test whether it is activated when animals use specific R-O associations. Having confirmed this pathway, we went on to test whether its activity is necessary for learning novel $\mathrm{R}-\mathrm{O}$ associations.

Subjects and apparatus. Twenty-six experimentally naive male LongEvans rats served as subjects. Sixteen (disconnection groups) received surgery to functionally disconnect the BLA and pDMS during behavioral training and a separate 10 (tracer group) received injections of the retrograde tracer fluoro-gold (FG) in the pDMS to examine direct inputs from the BLA. Animals were housed in groups of 2-3 and handled daily for 1 week before surgery or training. Training and testing took place in Med Associates operant chambers housed within sound- and light-resistant shells. Each chamber was equipped with two pumps, each of which was fitted with a syringe that delivered $0.1 \mathrm{ml}$ of fluid into a recessed magazine in the chamber. The two fluids were a $20 \%$ solution of sucrose or a $20 \%$ solution of polycose. The chambers were also equipped with a pellet dispenser that delivered one $45 \mathrm{mg}$ pellet when activated (Bio-Serv). The chambers contained two retractable levers that could be inserted to the left and the right of the magazine. A $3 \mathrm{~W}, 24 \mathrm{~V}$ houselight mounted on the wall across from the levers and magazine illuminated the chamber. Microcomputers equipped with MED-PC software (Med Associates) controlled the equipment and recorded responses.

Surgery. To enable functional disconnection of the BLA and pDMS during behavioral training, stereotaxic surgery was conducted under isofluorane anesthesia ( $5 \%$ induction; $1-2 \%$ maintenance) to lesion the BLA and implant cannulae in the pDMS. Rats were placed in a stereotaxic frame (Stoelting) and received a subcutaneous injection of $1 \mathrm{mg}$ of the local analgesic bupivacaine hydrochloride at the intended incision site. An incision was then made to expose the scalp and the incisor bar was adjusted to align bregma and $\lambda$ on the same horizontal plane and a small hole was drilled above the target regions. A 26 gauge guide cannula (Plastics One) targeting at the pDMS was implanted unilaterally (AP: -0.3 ; ML: \pm 2.6 ; DV: $-1.0 \mathrm{~mm}$ relative to bregma and dura for the DV coordinate; note that tips of the guide cannulae were positioned $3 \mathrm{~mm}$ dorsal to the intended infusion site: $-4.0 \mathrm{~mm}$ from dura) and fixed with dental acrylic. A unilateral BLA lesion was also produced by two unilateral infusions of $0.2 \mu \mathrm{l} 0.12 \mathrm{M}$ NMDA (AP: -2.3 and -3.0 ; ML: \pm 5.0 ; DV: $-7.0, \mathrm{~mm}$ from bregma and dura for the DV coordinate). Animals were divided into two groups. For animals in the ipsilateral group both the cannula and the lesion were placed in the same hemisphere thus leaving an intact BLA pathway in the opposite hemisphere. For the contralateral group the cannula and lesion were placed in opposite hemispheres thus, the BLA-pDMS was functionally disconnected in each hemisphere. The left or right position of the cannula and lesion was counterbalanced within each group.

To confirm direct active neural connections between BLA and pDMS, before training, a separate group of rats $(n=10)$ underwent stereotaxic surgery to inject FG into the pDMS. Rats were placed in a stereotaxic frame (Stoelting) and prepared as above with $100 \mathrm{~nL}$ of FG infused into the pDMS (AP: -0.3 ; ML: \pm 2.6 ; DV: $-4.0 \mathrm{~mm}$ relative to bregma and dura for DV). The injector was left in place for $5 \mathrm{~min}$ to allow for diffusion, and was then removed and the incision closed.

All rats were treated with $0.1 \mathrm{ml}$ of the analgesic caprofen and an intraperitoneal injection of $0.4 \mathrm{ml}(300 \mathrm{mg} / \mathrm{kg})$ solution of procaine penicillin and allowed at least 1 week of recovery before food restriction and training.

\section{Behavioral procedures}

Baseline instrumental training. Rats received one session of magazine training where polycose was delivered to the magazine according to a RT $60 \mathrm{~s}$ schedule. Rats were initially trained to respond on two levers to earn a $20 \%$ polycose solution. In an attempt to establish similar responding on the two levers, one lever was inserted at a time for $3 \mathrm{~min}$ and was then retracted and the other lever inserted for $3 \mathrm{~min}$. This sequence was repeated five times to comprise the $30 \mathrm{~min}$ session. The identity of the first lever was alternated across days. For the first two sessions, responding was reinforced on a continuous reinforcement schedule followed by $3 \mathrm{~d}$ of random ratio (RR)5, and finally $3 \mathrm{~d}$ of RR 10 reinforcement.

Specific $\mathrm{R}-\mathrm{O}$ training. On the next two days, two distinct rewards were introduced. Now, responding on one lever resulted in pellet delivery whereas responding on the other resulted in sucrose delivery according to a RR10 schedule of reinforcement. These sessions were also $30 \mathrm{~min}$ in length.

Reversal of $R-O$ associations under inactivation. For the final two days, the $\mathrm{R}-\mathrm{O}$-contingencies were reversed such that the lever that had earned pellets now earned sucrose and vice versa. Otherwise, the sessions were identical to those described above. For rats in the disconnection groups, $10 \mathrm{~min}$ before the beginning of both these sessions, these rats received an infusion of the GABA-A receptor agonist, muscimol (MUS; $0.1 \mathrm{~mm}$, Sigma-Aldrich) delivered via infusion cannulae (33 gauge; Plastics One) extending $3 \mathrm{~mm}$ below the guide cannula tip to inactivate the pDMS. A volume of $0.3 \mu \mathrm{l}$ was delivered to one hemisphere at a rate of $0.3 \mu \mathrm{l}$ per min by a syringe pump (Harvard Apparatus; PHD 22/2000).

Test for R-O learning using devaluation. Rats had ad libitum access to either pellets or sucrose for $1 \mathrm{~h}$ in separate feeding cages equipped with water bottles before a two-lever choice test conducted in extinction. The test session was 5 min long. Rats in the tracer group were killed $2 \mathrm{~h}$ after the beginning of this test, perfused, and their brains removed for immunohistochemistry. Rats in the disconnections groups received $1 \mathrm{~d}$ of retraining on the second set of selective $\mathrm{R}-\mathrm{O}$ associations under inactivation before a second test. This test was identical to the first except that the rats were pre-fed the opposite outcome.

Histology. At the end of the experiment, the rats were overdosed on sodium pentobarbital and perfused transcardially with a $4 \%$ paraformaldehyde solution in PBS ( $\mathrm{pH}, 7.2$; PFA). Brains were stored in PFA for a further $24 \mathrm{~h}$ and were then transferred to a $25 \%$ sucrose solution in PBS. Series of $30 \mu \mathrm{m}$ coronal sections were then taken and stored in $-20^{\circ} \mathrm{C}$ in cryoprotectant. For animals in the disconnection groups, tissue was stained using cresyl violet and slides were examined for placement of cannula and extent of the lesions, with the latter assessed by microscopically examining regions of marked cell loss and gliosis as well as shrinkage of a region relative to sham controls. Brains from animals in the tracer group underwent immunohistochemical processing as follows.

FG and fluorescent Nissl staining. Free floating sections from both the pDMS and BLA were stained for FG to confirm the placements of the injection site in pDMS and the retrograde labeled cells in the BLA. We additionally doubled-stained these sections with a fluorescent Nissl to better visualize regions of interest. Sections were given three $10 \mathrm{~min}$ washes in PBS followed by a $2 \mathrm{~h}$ incubation in a PBS solution containing $10 \%$ normal goat serum and $0.5 \%$ Triton $\mathrm{X}-100$. Sections were then incubated for $48 \mathrm{~h}$ at $4^{\circ} \mathrm{C}$ in the primary antibody rabbit anti-FG (1:3000; AB153, Millipore) in a PBS solution containing 2\% normal goat serum and $0.2 \%$ Triton X-100. Sections were again washed three times for 10 min each before being incubated for $90 \mathrm{~min}$ in a PBS solution containing $2 \%$ normal goat serum, $0.2 \%$ Triton X-100, a goat anti-rabbit Alexa Fluor 488-conjugated secondary antibody (1:1000; A11008, Invitrogen) and a fluorescent Nissl stain (1:50; N21483, Invitrogen). Sections were 
A

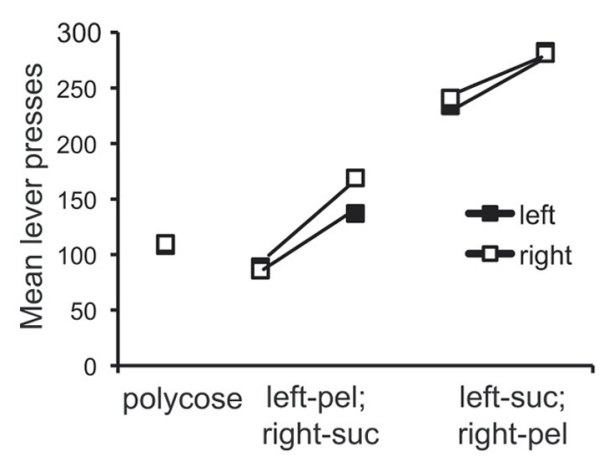

B

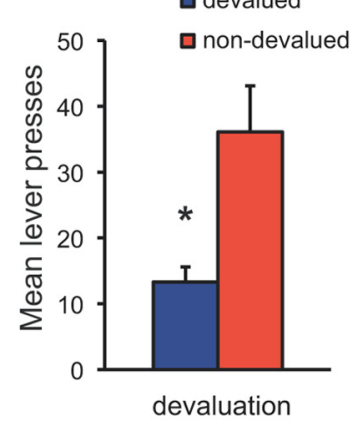

C

$\begin{array}{ll}\text { Measure } & \text { Mean (SE) } \\ \text { Total c-Fos } & 81.6(13.4) \\ \text { Total FG } & 406.4(45) \\ \text { Double-labelled } & 20.5(4.2) \\ \begin{array}{ll}\text { Double as a \% of } \\ \text { total c-Fos }\end{array} & 28.9(4.4)\end{array}$

Training Phase

Figure 1. A, Mean lever presses for the left and right lever for the final day of training with a common reward (polycose), with the introduction of novel R-0 contingences (left lever-pellet; right lever-sucrose), and the reversal of those contingencies (left lever-sucrose; right lever-pellets). $\boldsymbol{B}$, Mean lever presses following devaluation by specific satiety. Devalued refers to responding on the lever most recently paired with the pre-fed outcome. Non-devalued refers to responding on the lever most recently earning the alternate outcome. Asterisk indicates that responding on the devalued lever differed significantly from responding on the non-devalued lever, $p<0.05$. C, Mean ( \pm SE) c-Fos-positive, FG-positive, and double-labeled cells in the BLA following devaluation testing.

then washed a final three times in PBS before being mounted on slides and coverslipped with Fluoromount-G (Southern Biotech).

c-Fos immunoreactivity. Additional sections containing the BLA underwent processing to identify cells that were positive for (1) c-Fos immunoreactivity (2) retrograde transport of FG (3) and coexpression of c-Fos in FG-labeled cells. Similar to the procedures described above, sections were rinsed three times for $10 \mathrm{~min}$ in PBS and transferred to PBS solution with $0.5 \%$ Triton X-100 and $10 \%$ normal goat serum for $2 \mathrm{~h}$. Sections were then incubated in a rabbit anti-c-Fos antibody (1:500; sc-52, Santa Cruz Biotechnology) with 0.2\% Triton X-100 and 2\% normal goat serum in PBS for $48 \mathrm{~h}$ at $4^{\circ} \mathrm{C}$. Three $10 \mathrm{~min}$ PBS washes were given before sections were placed in a goat anti-rabbit Alexa Flour 488conjugated secondary antibody (1:1000; Invitrogen) with $0.2 \%$ Triton $\mathrm{X}-100$ and $2 \%$ normal goat serum in PBS for $90 \mathrm{~min}$. A final three $10 \mathrm{~min}$ PBS washes were then given before sections were mounted on slides and coverslipped with Fluoromount-G (Southern Biotech).

Fluorescent imaging and quantification of immunoreactive cells. The photomicrographs of the FG injection site in pDMS and projections from the BLA (see Fig. $2 A, B$ ) were composites of multiple $10 \times$ images stitched together. Images were taken from an Olympus FV10i confocal microscope. Higher resolution images of the c-Fos and FG in the BLA for quantification (see Fig. 2D,E) were acquired with an Olympus BX61WI confocal microscope. All neurons expressing c-Fos, FG, and c-Fos colocalized with FG were counted by an observer blind to the experimental conditions using ImageJ (rsb.info.nih.gov/ij).

\section{Experiment 2: the role of the BLA-pDMS pathway in the expression of $\mathrm{R}-\mathrm{O}$ associations}

Experiment 1 evaluated whether a functional connection between the BLA and pDMS is necessary for the acquisition of novel R-O associations. The aim of Experiment 2 was to test whether this pathway is necessary for the expression of $\mathrm{R}-\mathrm{O}$ associations in performance once formed.

Subjects and apparatus. Seventeen experimentally naive male LongEvans rats (Monash) served as subjects. The housing conditions and training apparatus were identical to those described above.

Surgery. Stereotaxic surgery was conducted under isoflurane anesthesia to implant guide cannulae targeted at the pDMS and to produce cell body lesions of the BLA using methods identical to those described in Experiment 1. Again, rats were assigned to either ipsilateral or contralateral groups and thus received cannula implants and lesions in the same or opposite hemispheres, respectively.

\section{Behavioral procedures}

Methods for magazine and baseline instrumental training with polycose were identical to those above. To match training history and to allow comparison with Experiments 1 and 2, similar specific $\mathrm{R}-\mathrm{O}$ training was also used. Rats underwent $2 \mathrm{~d}$ of training in which one lever earned pellets and the other sucrose. These relationships were then reversed and the rats underwent two additional days of training before devaluation testing.

Devaluation tests. Rats were given $1 \mathrm{~h}$ of ad libitum access to either sucrose or pellets and then received an infusion of MUS $(0.3 \mu \mathrm{l}$ of 0.1 $\mathrm{mm}) 10 \mathrm{~min}$ before the beginning of a 5 min extinction test. Rats underwent one retraining session on the second pair of R-O associations (without inactivation) before a second devaluation test. Rats were pre-fed the opposite outcome from the first test and again received an infusion of MUS $10 \mathrm{~min}$ before the $5 \mathrm{~min}$ extinction test.

Consumption tests of specific satiety. To assess the ability of the rats to distinguish between the two outcomes following inactivation, they were given a consumption version of the specific-satiety devaluation test. One of the two outcomes was devalued in the same manner as in the tests described above, but now the dependent measure used was consumption of either that same outcome or a different outcome. Normal performance on this test (i.e., decreased consumption of the same food item relative to a different food) requires that the animals can distinguish between the two food items. The animals received two tests. In each test, they received $1 \mathrm{~h}$ of ad libitum access to one of the foods, either pellets or sucrose, in a feeding cage. The pre-fed food was removed, and the animal received an infusion of muscimol. Then the animals were returned to the feeding cage and presented with the test food, which was sucrose for all animals, for an additional $20 \mathrm{~min}$, and their consumption in that test period was recorded. In each test, half of the animals received the same food in prefeeding and testing (sucrose-sucrose) and for the remaining animals the food was different between prefeeding and testing (pelletssucrose). The following day, the rats underwent a second test which was identical except that they were pre-fed the opposite food. If devaluation was effective, we predicted that rats would consume more sucrose following pellet prefeeding than following sucrose prefeeding.

Histology. At the end of the experiment tissue was prepared and examined as in Experiment 2 to determine the placement of the lesions and cannulae tips.

\section{Results}

Experiment 1: pretraining disconnection of the BLA and pDMS prevents the acquisition of novel $\mathrm{R}-\mathrm{O}$ associations

\section{Tracer group}

Baseline instrumental training. Lever-press performance on the final day of training with the common outcome (polycose) is shown in Figure $1 A$, lef. Rats responded equally on the two levers in this phase of training $\left(F_{(1,9)}=0.4, p>0.05\right)$.

Specific $\mathrm{R}-\mathrm{O}$ training. As shown in Figure $1 A$, the rats continued to respond when the novel outcomes were introduced. Responding increased across days $\left(F_{(1,9)}=16.4, p<0.01\right)$ but there 

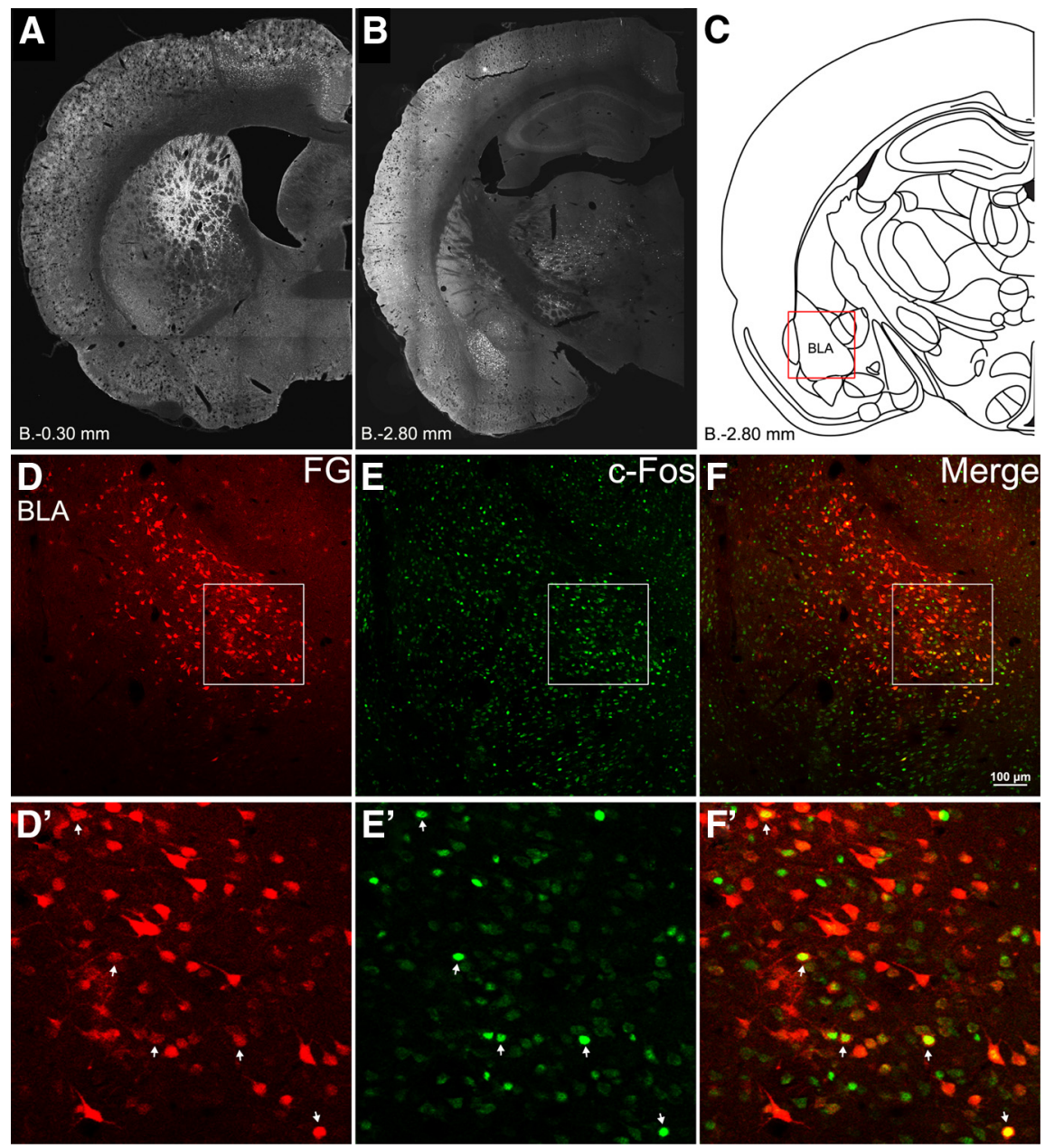

Figure 2. $\quad \boldsymbol{A}, \mathrm{A}$ representative example of the $\mathrm{FG}$ injection site in the pDMS and the pattern of $F G$ that was retrogradely transported to the $B L A(B)$. Panel C shows the region of the BLA that is magnified in panels D-Fbelow. $\boldsymbol{D}$, FG positive cells in the BLA The white inset indicates the region that is further magnified in panels $D^{\prime}-F^{\prime} . E$, c-Fos positive cells in the BLA. $\boldsymbol{F}$, Coexpression of $F G$ and c-Fos in the BLA. (D'-F') Magnification of panels D-F above.

was no difference in responding on the two levers $\left(F_{(1,9)}=0.08\right.$, $p>0.05)$.

\section{Reversal of $\mathrm{R}-\mathrm{O}$ associations}

The rats continued to show robust responding when the $\mathrm{R}-\mathrm{O}$ contingencies were reversed (Fig. $1 A$, right) and increased responding across days $\left(F_{(1,9)}=5.4, p<0.05\right)$ but still showed no difference in responding on the two levers $\left(F_{(1,9)}=0.01, p>\right.$ $0.05)$.

Devaluation tests. As shown in Figure 1B, prefeeding one of the previously earned outcomes, was effective in selectively reducing responding. This was reflected as a significant effect of devaluation $\left(F_{(1,9)}=8.7, p<0.05\right)$. The performance of these rats provides evidence that following this training regimen, intact rats use the most recently learned $\mathrm{R}-\mathrm{O}$ relationships to direct performance. This may be somewhat surprising given previous reports that when a response is trained sequentially with multiple outcomes, both $\mathrm{R}-\mathrm{O}$ associations contribute equally to performance (Rescorla, 1995, 1996b). There are many procedural differences between the present studies and those by Rescorla including the use of ratio versus interval schedules, devaluation produced by specific-satiety versus conditioned taste aversion, and the training of two versus four responses. Notably, in Rescorla's studies, training in each phase was longer (8-10 d vs 2) and devaluation was achieved using conditioned taste aversion (taking $4 \mathrm{~d}$ ). The longer training may not only have resulted in a stronger initial association more likely to persist at test, but the substantial delay between the end of initial R-O training and testing $(\sim 14 \mathrm{~d}$ compared with $3 \mathrm{~d}$ in our design) provides an opportunity for the initial association to recover (Rescorla, 1996a). The original associations may well be maintained in our paradigm but further experiments are needed to examine whether they can be revealed (e.g., by allowing greater opportunity for spontaneous recovery). However, what is clear is that with the training and testing parameters used in the current study, animals tend to rely more strongly on the most recently acquired $\mathrm{R}-\mathrm{O}$ associations to direct performance, which is important for interpreting the subsequent results in the disconnection groups.

\section{FG and c-Fos immunoreactivity in the BLA}

We combined retrograde tracing with FG with a marker of cellular activation, c-Fos, to identify activation of BLA afferents to the $\mathrm{pDMS}$ during devaluation testing. The pattern of retrograde transport of FG to the BLA following injection into the pDMS is shown in Figure $2(A$, injection site; $B, D, D^{\prime}$, labeling in the BLA). As is clear from the figure, injection of FG into this medial and posterior region of the striatum led to fairly robust labeling in the BLA. We examined c-Fos as evidence of neuronal activation after rats underwent a choice extinction test following devaluation and found evidence of activation in the BLA (Fig. $2 E, E^{\prime}$ ). Figure $2 F, F^{\prime}$ show cells positive for both c-Fos and FG. The counts of c-Fos positive, FG positive, and double-labeled cells are summarized in Figure $1 C$. As we were most interested in c-Fos activation in cells projecting to the pDMS (i.e., the doublelabeled cells), to account for any differences in overall levels of c-Fos, we considered the percentage of c-Fos positive cells that were also positive for FG. We conducted correlational analyses (Pearson correlation) examining the relationship between the number of double labeled cells as a percentage of total c-Fos positive cells and the magnitude of the devaluation effect (i.e., number of lever-presses on the non-devalued lever minus the number of lever presses on the devalued lever). We found that these measures were significantly correlated $(r=0.77, p<0.01)$. Together these data confirm a direct pathway between BLA and the region of the pDMS previously shown to be critical for $\mathrm{R}-\mathrm{O}$ learning and demonstrate that this pathway is active during a choice extinction test, implicating a functional role for this pathway in mediating utilization of information about selective $\mathrm{R}-\mathrm{O}$ associations. The necessity of this pathway was evaluated with disconnection procedures, the results of which follow.

\section{Disconnection groups}

Histology. No recovery problem or weight loss was observed after surgery. Figure 3 displays the maximum and minimum damage 
resulting from the lesions of the BLA (right) and cannula placement within the pDMS (left) for the rats included in the behavioral analyses for Experiments 2 and 3 based on the stereotaxic atlas of the rat brain by Paxinos and Watson (1998). Rats with damage or placement outside the target region were excluded from the behavioral analyses. BLA lesions typically created damage throughout the majority of the rostrocaudal extent of the BLA with some sparing of tissue in the caudal BLA and included the basal, accessory basal, and lateral divisions of the region. There were no notable differences in the extent of the lesions or cannula placements for the two groups apart from their location in either the ipsilateral or contralateral hemispheres.

Baseline instrumental training. All rats learned to lever-press for the polycose solution. Mean response rates for left and right lever for the final training session are shown in Figure 4A. As shown in the figure, the rats showed similar performance on the left and right levers $\left(F_{(1,14)}=0.01\right.$, $p>0.05)$ and performance was similar across groups (no effect of group; $F_{(1,14)}=$ $0.1,>.05$; no group $\times$ lever interaction; $\left.F_{(1,14)}=0.06, p>0.05\right)$.

\section{Specific $R-O$ training}

As shown in Figure $4 A$ when the pellet and sucrose rewards were introduced, the rats continued to show robust and even responding on the two levers $\left(F_{(1,14)}=0.03, p>0.05\right]$ which increased across days $\left(F_{(1,14)}=6.04, p<0.05\right)$. Performance remained similar across groups (no effect of group; $F_{(1,14)}=0.1$, $p>0.05$; no group $\times$ lever interaction; $F_{(1,14)}=0.21, p>0.05$ and no interactions with day; Fs $<1$ ).

\section{Reversal of $\mathrm{R}-\mathrm{O}$ associations under inactivation}

As shown in Figure $4 A$, the rats maintained robust responding when the selective $\mathrm{R}-\mathrm{O}$ associations were reversed following inactivation increasing responding across days $\left(F_{(1,14)}=29.9, p<\right.$ 0.01 ) and continuing to respond similarly on the two levers (no effect of lever; $\left.F_{(1,14)}=0.14, p>0.05\right)$. There remained no effect of group $\left(F_{(1,14)}=0.18, p>0.05\right)$ and no interactions with this factor $(\mathrm{Fs}<1)$.

\section{Test for R-O learning using devaluation}

Inspection of Figure $4 B$ suggests that the prefeeding treatment had different effects in the two groups. Although each group selectively reduced responding on one lever following the devaluation treatment, they differed with regard to on which lever they showed this effect. Whereas the ipsilateral group showed the expected pattern of responding, reducing responding on the lever most recently paired with the devalued outcome, the contralateral group decreased performance on the opposite lever. This pattern would be expected if their performance was based on the original $\mathrm{R}-\mathrm{O}$ relationships to which they were exposed before reversal and suggests that disconnection of the BLA-pDMS path-

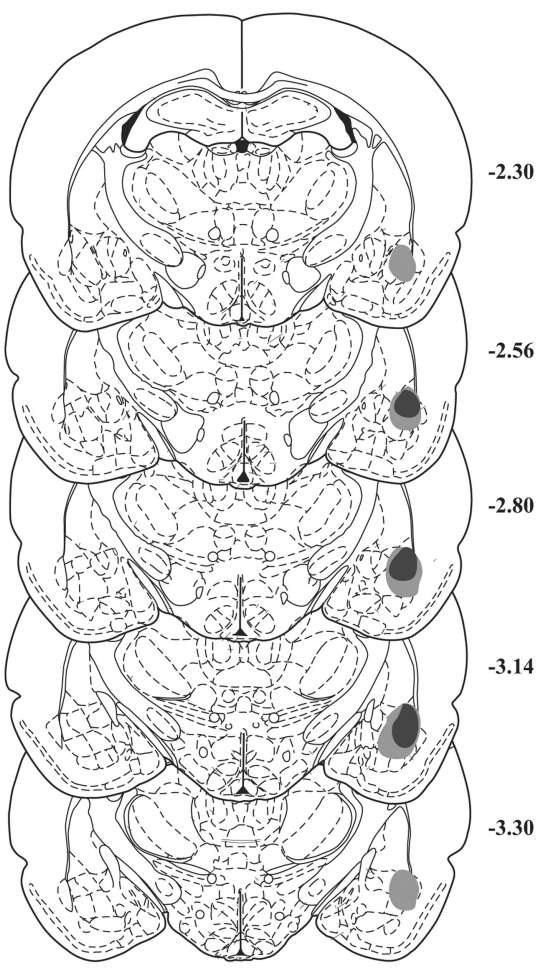

Figure 3. Schematic representation of lesions to the BLA (right) and cannula placements in the pDMS (left) for rats in Experiments 2 and 3 . Note that for rats in the ipsilateral groups, lesions and cannula were in the same hemisphere whereas for rats in the relative to bregma, with some subjects showing evidence of damage slightly further rostrally or caudally, and typically included the medial regions as well in the most caudal areas of the BLA. Cannula were positioned in the pDMS striatum typically between +0.2 to $-0.3 \mathrm{~mm}$ from bregma. Numbers indicate distance $(\mathrm{mm})$ from bregma. Based on Paxinos and Watson, 1998.

way before the reversal of the $\mathrm{R}-\mathrm{O}$ contingencies prevented the formation of the new associations. Initial analyses found that the devaluation effects in the two groups were similar regardless of whether the animals were pre-fed pellets or sucrose (no effect of devalued outcome; $F_{(1,14)}=0.89, p>0.05$; ipsilateral group: 18.7/30.2 and 15.6/27.6 for devalued/non-devalued responses when pellets or sucrose were devalued, respectively; contralateral group: 22.8/12.9 and 20.2/10.6 for devalued/non-devalued responses when pellets or sucrose were devalued, respectively) and so the data were collapsed across this variable in subsequent analyses. The statistical analyses found no effect of devaluation $\left(F_{(1,14)}=2.2\right.$, $p>0.05$ ] or of group; $\left.F_{(1,14)} 1.7, p>0.05\right)$ but there was a significant interaction between group and the devaluation treatment $\left(F_{(1,14)}=\right.$ $32.6, p<0.01)$. Simple effects analyses found a significant difference in performance in both the contralateral $\left(F_{(1,8)}=14.7, p<0.01\right)$ and ipsilateral $\left(F_{(1,6)}=16.4, p<0.01\right)$ groups confirming the effective but opposite devaluation effects in the two groups.

\section{Experiment 2: disconnection of the BLA and pDMS} immediately before testing prevents the expression of specific $\mathrm{R}-\mathrm{O}$ associations

Baseline instrumental training. All rats learned to respond on both levers for the common reward (Fig. $5 A$ ). There was no effect of lever $\left(F_{(1,15)}=1.1, p>0.05\right)$, no effect of group $\left(F_{(1,15)}=0.3, p>0.05\right)$, and no interaction between these factors $\left(F_{(1,15)}=0.07, p>0.05\right)$.

Specific $R-O$ training. As shown in Figure $5 A$, responding increased across days with the introduction of the novel rewards $\left(F_{(1,15)}=11.9, p<0.01\right)$ but remained similar for the two levers 
A
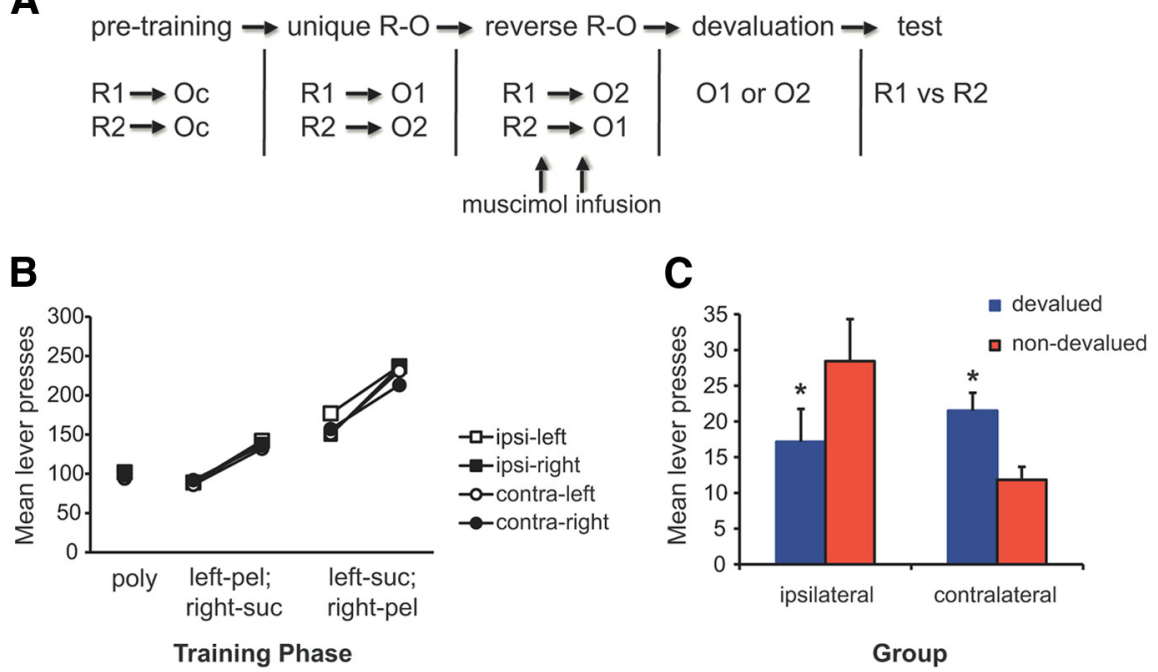

Figure 4. A, Schematic representation of the experimental design for Experiment 1. Rats underwent surgery to lesion the BLA and implant cannulae targeting the pDMS. They were then trained to perform two instrumental responses, each earning a common reward (polycose). The next underwent two training sessions in which each response earned a unique outcome. These R- 0 contingencies were reversed in two subsequent sessions and critically, the pDMS was inactivated before these sessions. One of the two instrumental outcomes was then devalued with a specific satiety procedure before a 5 min extinction test. $\boldsymbol{B}$, Mean lever presses for the left and right lever for rats in the ipsilateral (ipsi) and contralateral (contra) groups on the final day of training with a common reward (polycose), with the introduction of novel R-0 contingences (left lever-pellet; right lever-sucrose), and the reversal of those contingencies (left lever-sucrose; right lever-pellets). Note that rats received infusions of muscimol before the last two training sessions. C, Mean lever presses following devaluation by specific satiety. Devalued refers to responding on the lever most recently paired with the pre-fed outcome. Non-devalued refers to responding on the lever most recently earning the alternate outcome. Asterisk indicates that responding on the devalued lever differed significantly from responding on the nondevalued lever, $p<0.05$. There were no infusions before testing.

$\left(F_{(1,15)}=1.7, p>0.05\right)$ and two groups $\left(F_{(1,15)}=0.3, p>0.05\right)$. There were no interactions among these factors (all Fs $<1$ ).

Reversal of $\mathrm{R}-\mathrm{O}$ associations. As shown in Figure 5A responding continued to increase across days $\left(F_{(1,15)}=17.6, p<0.01\right)$ but remained similar for the two levers $\left(F_{(1,15)}=0.01, p>0.05\right)$ and two groups $\left(F_{(1,15)}=0.3, p>0.05\right)$. No interactions were significant (all Fs $<1$ ).

Test for expression of $\mathrm{R}-\mathrm{O}$ associations following inactivation using devaluation. The test data suggest that the BLA-pDMS pathway is also needed for selective performance following outcome devaluation. As shown in Figure 5B, rats in the ipsilateral group decreased performance of the response most recently associated with the currently devalued outcome. In contrast, rats in the contralateral group failed to show a selective devaluation effect responding at similar rates on the two levers. Initial analyses found that the devaluation effects in the two groups were similar regardless of whether the animals were pre-fed pellets or sucrose (no effect of devalued outcome; $F_{(1,15)}=1.15, p>0.05$; ipsilateral group: $11.5 / 17.25$ and 9.3/25.6 for devalued/non-devalued responses when pellets or sucrose were devalued, respectively; contralateral group: 20.6/25.2 and 14.7/15.8 for devalued/nondevalued responses when pellets or sucrose were devalued, respectively) and so the data were collapsed across this variable in subsequent analyses. This description was confirmed by the statistical analysis which found an overall effect of devaluation $\left(F_{(1,15)}=21.9, p<0.01\right)$ and, although there was no overall effect of group $\left(F_{(1,15)}=1.3, p>0.05\right)$, an interaction between these factors $\left(F_{(1,15)}=7.6, p<0.05\right)$. Simple effects analyses found a significant devaluation effect in the ipsilateral $\left(F_{(1,7)}=20.9, p<\right.$ $0.01)$ but not contralateral $\left(F_{(1,8)}=2.5, p>0.05\right)$ group.

Consumption tests. It is possible that inactivation before testing interfered with the rats' ability to distinguish between the two outcomes or to recall the updated value of the pre-fed outcome which would prevent selective lever-press performance. To establish whether the disconnection treatment reduced the effectiveness of the devaluation treatment rather than the ability to use this information to direct selective performance, we conducted an additional devaluation test where consumption rather than lever-pressing was the dependent measure. The results show that rats in both groups consumed less when the test food was the same as the pre-fed food compared with when the pre-fed and test foods were different (Fig. 6). There was a significant effect of devaluation $\left(F_{(1,15)}=32.7, p<0.01\right)$, no effect of group $\left(F_{(1,15)}<1\right)$, and no interaction between these factors $\left(F_{(1,15)}<\right.$ $1)$. This result indicates that the disconnection treatment did not reduce the efficacy of the devaluation treatment per se, but rendered rats unable to use the updated value of the outcome to direct performance.

\section{Discussion}

The current data demonstrate that a functional connection between the BLA and pDMS contributes importantly to instrumental learning and performance. We found that when this BLA-pDMS connection was disrupted before sessions in which novel R-O relationships were introduced rats failed to learn about the new specific $\mathrm{R}-\mathrm{O}$ contingencies, despite normal performance during these sessions. Instead the rats appeared to rely on previously established associations to control performance in a devaluation test. Similarly, when the BLA-pDMS pathway was disconnected immediately before devaluation testing, rats failed to show selective performance in the test indicating that they were unable to retrieve specific $\mathrm{R}-\mathrm{O}$ associations after inactivation of the pDMS contralateral to the BLA lesion.

These data add to reports emphasizing the importance of BLA inputs to the cortical and striatal regions in controlling aspects of reward-related learning and performance. For example, Shiflett and Balleine (2010) found that disconnection of the BLA from the nucleus accumbens core, impaired sensitivity of instrumental performance to outcome devaluation whereas disconnection from accumbens shell removed the excitatory influence of specific reward-related cues in a Pavlovian-instrumental transfer paradigm. This is consistent with demonstrations that input from the BLA is necessary for NAc neurons to develop neural responses in relation to presentations of reward-associated stimuli (Ambroggi et al., 2008; Jones et al., 2010). In general, the BLA seems essential for selective performance on tasks that require the use of sensory-specific outcome representations or the association of these representations with specific responses or stimuli (Hatfield et al., 1996; Blundell et al., 2001, Corbit and Balleine, 2005; Dwyer and Killcross, 2006). As such, it is not surprising that inputs from the BLA to multiple structures are important for selective performance in a variety of tasks that require detailed information about distinct outcomes. The present study provides evidence that the input from the BLA to the pDMS provides information 
about the unique sensory and motivational properties of different outcomes needed for the $\mathrm{pDMS}$ to establish selective R-O associations (Yin et al., 2005; Corbit and Janak, 2010). The fact that, like the NAc core, it influences performance based on these associations suggests that these structures may interact to influence aspects of the selection and execution of actions based on outcome evaluation but whether these structures control distinct aspects of that process remains to be determined.

The deficits reported here are not easily accounted for by nonspecific impairment in motor output, as ipsilateral and contralateral groups showed similar levels of performance when they received the inactivation treatment before training sessions. Further, these results cannot be explained by an inability of the rats in the contralateral groups to discriminate between the two outcomes following disconnection of the BLA-pDMS pathway; when we evaluated their sensitivity to devaluation by measuring consumption of either the same or a different outcome to that on which the animals had been prefed, disconnection was without effect and these rats showed a selective devaluation effect. Normal performance in a consumption version of the devaluation task confirms that the disconnection treatment immediately before testing did not interfere with the effectiveness of the devaluation treatment itself or the ability of rats to discriminate between the alternative outcomes. Thus, our data indicate that the BLA-pDMS pathway is needed for information about unique outcomes to be integrated with specific responses allowing both the acquisition of selective associations and, subsequently, the ability to use these associations to direct performance.

Previous reports have suggested that the DMS, particularly cholinergic activity in the DMS, is involved in reversal learning (Brown et al., 2010; Bradfield et al., 2013). Thus, it is possible that inactivation of this structure before sessions in which the $\mathrm{R}-\mathrm{O}$ contingency was reversed impaired behavioral flexibility and the ability of rats to reverse previously learned $\mathrm{R}-\mathrm{O}$ contingencies rather than the ability to acquire a specific $\mathrm{R}-\mathrm{O}$ association. Although experiments where inactivation occurs during the initial introduction of novel $\mathrm{R}-\mathrm{O}$ associations without reversal could specifically rule out this possibility, it should be noted that manipulations of either the pDMS or BLA abolish new (nonreversed) action-outcome learning, which is not true of cholinergic manipulations in the DMS. Furthermore, inactivation of the pDMS before the introduction of novel R-O associations has confirmed that it is essential for R-O learning with this simpler design. Hence, the effects we report are not specific to reversal learning (Corbit and Janak, 2010). Furthermore, although impaired reversal could account for the pattern of results in Experiment 1 , the fact that the rats failed to acquire the new actionoutcome associations but were capable of retaining and using the previously acquired associations to guide their choice performance suggests the deficit on test was not due simply to a general loss of pDMS function. Such a conclusion could not be drawn if inactivation occurred during the initial introduction

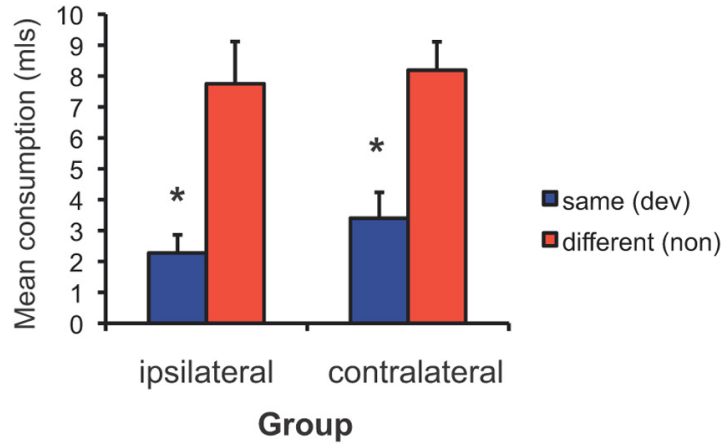

Figure 6. Mean milliliters of sucrose consumed following $1 \mathrm{~h}$ ad libitum access to either sucrose (same; devalued) or pellets (different; non-devalued). Note that the rats received an infusion of muscimol after pre-feeding and before introduction of the test food. Asterisk indicates that consumption following prefeeding of the same outcome (devalued) differed from that following prefeeding of a different outcome (non-devalued), $p<0.05$.

of novel R-O associations. Finally, a deficit specific to reversal does not readily explain the results of Experiment 2 where disconnection of the BLA-pDMS pathway occurred immediately before testing after the reversal was complete.

The observed deficits produced by disconnection of the BLApDMS pathway do not rule out possible indirect connections between these structures or a role for other brain areas. For example, the DMS receives afferents from the prelimbic cortex (PL; Berendse et al., 1992), a region that has been shown in the rat to be critical for the acquisition of goal-directed behaviors (Corbit and Balleine, 2003; Ostlund and Balleine, 2005) that also receives inputs from the BLA (Hoover and Vertes, 2007). However, disconnection of the BLA and PL has been reported to be without effect on goal-directed performance following devaluation 
(Coutureau et al., 2009) and thus an indirect pathway including the PL is unlikely to account for the current results. The DMS also receives input from the orbitofrontal cortex (OFC) which projects broadly through the striatum in a topographic manner (Schilman et al., 2008) and that is itself highly interconnected with the BLA (Krettek and Price, 1978; McDonald, 1991) and could provide an indirect pathway through which the learningrelated activity of the BLA might influence the DMS. However, despite a role in Pavlovian valuation tasks (Schoenbaum et al., 2011; Rudebeck et al., 2013) the role of the OFC in relation to instrumental performance is best characterized as controlling the influence of reward-predictive stimuli on response choice rather than mediating performance based on outcome value (Ostlund and Balleine, 2007; Balleine et al., 2011) and is also, therefore, unlikely to account for the deficits observed. The BLA also makes strong connections with the gustatory region of insular cortex and these connections have recently been found to be necessary for the retrieval of outcome value to guide choice performance after changes in value, such as those induced by outcome devaluation (Parkes and Balleine, 2013). However, the insular cortex maintains far stronger connections with the accumbens core than the pDMS and, therefore, has appeared a more likely candidate to control performance of goal-directed actions rather than the acquisition of action-outcome associations. Further, our data from the tracer group in Experiment 1 confirmed direct neural connections between the BLA and pDMS and demonstrated that this pathway is indeed activated at least during the test of actionoutcome association in Experiment 1. Although this does not rule out a role for other pathways, it does demonstrate that a disruption of a direct pathway could plausibly account for the observed deficits. Whether the connections are direct or indirect, clearly, transfer of information from BLA to pDMS is needed for selective instrumental learning and the maintenance of that information in the intact pDMS for instrumental performance.

The current data complement the recent report that a different amydgalostriatal circuit involving the central nucleus of the amygdala (CN) and the dorsolateral striatum (DLS) is essential for establishing response habits (Lingawi and Balleine, 2012). In that study rats that received either bilateral lesions of the $\mathrm{CN}$ or lesions that functionally disconnected the CN and DLS before overtraining of a lever-press response, remained sensitive to devaluation when controls showed clear evidence of habitual performance. Whereas this connection is likely to be indirect, via the thalamus (Sah et al., 2003), or perhaps more likely, the substantia nigra (Beckstead et al., 1979; Gonzales and Chesselet, 1990), these data nonetheless demonstrate that, functionally, interaction between CN and DLS is vital for normal habit learning. Together these data suggest, therefore, that, in addition to previous dissociations within the dorsal striatum, parallel amygdalostriatal circuits contribute to goal-directed and habitual control of instrumental performance.

In summary, these results demonstrate that a projection from the BLA to the pDMS, a structure previously shown to be essential for R-O learning, exists and is activated when rats must rely of specific $\mathrm{R}-\mathrm{O}$ associations to inform behavioral choice. Furthermore, functional disconnection of this pathway using asymmetric lesion and inactivation techniques before training sessions disrupts the acquisition of new R-O associations. Finally, functional disconnection of this pathway immediately before devaluation testing disrupts selective performance based on specific $\mathrm{R}-\mathrm{O}$ associations confirming an important role for a BLA-pDMS pathway in goal-directed learning and performance.

\section{References}

Alexander GE, DeLong MR, Strick PL (1986) Parallel organization of functionally segregated circuits linking basal ganglia and cortex. Annu Rev Neurosci 9:357-381. CrossRef Medline

Ambroggi F, Ishikawa A, Fields HL, Nicola SM (2008) Basolateral amygdala neurons facilitate reward-seeking behavior by exciting nucleus accumbens neurons. Neuron 59:648-661. CrossRef Medline

Balleine BW, Killcross AS, Dickinson A (2003) The effect of lesions of the basolateral amygdala on instrumental conditioning. J Neurosci 23:666675. Medline

Balleine BW, Leung BK, Ostlund SB (2011) The orbitofrontal cortex, predicted value, and choice. Ann N Y Acad Sci 1239:43-50. CrossRef Medline

Balleine BW, Killcross S (2006) Parallel incentive processing: an integrated view of amygdala function. Trends Neurosci 29:272-279. CrossRef Medline

Beckstead RM, Domesick VB, Nauta WJ. (1979) Efferent connections of the substantia nigra and ventral tegmental area in the rat. Brain Res 175:191217. CrossRef Medline

Berendse HW, Galis-de Graaf Y, Groenewegen HJ (1992) Topographical organization and relationship with ventral striatal compartments of prefrontal corticostriatal projections in the rat. J Comp Neurol 316:314-347. CrossRef Medline

Blundell P, Hall G, Killcross S (2001) Lesions of the basolateral amygdala disrupt selective aspects of reinforcer representation in rats. J Neurosci 21:9018-9026. Medline

Bradfield LA, Bertran-Gonzalez J, Chieng B, Balleine BW (2013) The thalamo-striatal pathway and the cholinergic control of goal-directed action: interlacing new and existing action-outcome associations in the striatum. Neuron 79:153-166. CrossRef Medline

Brown HD, Baker PM, Ragozzino ME (2010) The parafascicular thalamic nucleus concomitantly influences behavioral flexibility and dorsomedial striatal acetylcholine output in rats. J Neurosci 30:14390-14398. CrossRef Medline

Corbit LH, Balleine BW (2003) The role of prelimbic cortex in instrumental conditioning. Behav Brain Res 146:145-157. CrossRef Medline

Corbit LH, Balleine BW (2005) Double dissociation of basolateral and central amygdala lesions on the general and outcome-specific forms of Pavlovian-instrumental transfer. J Neurosci 25:962-970. CrossRef Medline

Corbit LH, Janak PH (2010) Posterior dorsomedial striatum is critical for both selective instrumental and Pavlovian reward learning. Eur J Neurosci 31:1312-1321. CrossRef Medline

Coutureau E, Marchand AR, Di Scala G (2009) Goal-directed responding is sensitive to lesions to the prelimbic cortex or basolateral nucleus of the amygdala but not to their disconnection. Behav Neurosci 123:443-448. CrossRef Medline

Dwyer DM, Killcross S (2006) Lesions of the basolateral amygdala disrupt conditioning based on the retrieved representations of motivationally significant events. J Neurosci 26:8305-8309. CrossRef Medline

Gonzales C, Chesselet MF (1990) Amygdalonigral pathway: an anterograde study in the rat with Phaseolus vulgaris leucoagglutinin (PHA-L). J Comp Neurol 297:182-200. CrossRef Medline

Haber SN, Kim KS, Mailly P, Calzavara R (2006) Reward-related cortical inputs define a large striatal region in primates that interface with associative cortical connections, providing a substrate for incentive-based learning. J Neurosci 26:8368-8376. CrossRef Medline

Hatfield T, Han JS, Conley M, Gallagher M, Holland P (1996) Neurotoxic lesions of basolateral, but not central, amygdala interfere with Pavlovian second-order conditioning and reinforcer devaluation effects. J Neurosci 16:5256-5265. Medline

Hoover WB, Vertes RP (2007) Anatomical analysis of afferent projections to the medial prefrontal cortex in the rat. Brain Struct Funct 212:149-179. CrossRef Medline

Johnson AW, Gallagher M, Holland PC (2009) The basolateral amygdala is critical to the expression of Pavlovian and instrumental outcome-specific reinforcer devaluation effects. J Neurosci 29:696-704. CrossRef Medline

Jones JL, Day JJ, Aragona BJ, Wheeler RA, Wightman RM, Carelli RM (2010) Basolateral amygdala modulates terminal dopamine release in the nucleus accumbens and conditioned responding. Biol Psychiatry 67:737-744. CrossRef Medline

Kelley AE, Domesick VB, Nauta WJ (1982) The amygdalostriatal projection 
in the rat-an anatomical study by anterograde and retrograde tracing methods. Neuroscience 7:615-630. CrossRef Medline

Krettek JE Price JL (1978) Amygdaloid projections to subcortical structures within the basal forebrain and brainstem in the rat and cat. J Comp Neurol 178:225-254. CrossRef Medline

Lingawi NW, Balleine BW (2012) Amygdala central nucleus interacts with dorsolateral striatum to regulate the acquisition of habits. J Neurosci 32:1073-1081. CrossRef Medline

McDonald AJ (1991) Topographical organization of amygdaloid projections to the caudatoputamen, nucleus accumbens, and related striatal-like areas of the rat brain. Neuroscience 44:15-33. CrossRef Medline

Ostlund SB, Balleine BW (2005) Lesions of medial prefrontal cortex disrupt the acquisition but not the expression of goal-directed learning. J Neurosci 25:7763-7770. CrossRef Medline

Ostlund SB, Balleine BW (2007) Orbitofrontal cortex mediates outcome encoding in Pavlovian but not instrumental conditioning. J Neurosci 27:4819-4825. CrossRef Medline

Parent A, Hazrati LN (1995) Functional anatomy of the basal ganglia: I. The cortico-basal ganglia-thalamo-cortical loop. Brain Res Brain Res Rev 20: 91-127. CrossRef Medline

Parkes SL, Balleine BW (2013) Incentive memory: evidence the basolateral amygdala encodes and the insular cortex retrieves outcome values to guide choice between goal-directed actions. J Neurosci 33:8753-8763. CrossRef Medline

Paxinos G, Watson C (1998) The rat brain in stereotaxic coordinates. San Diego: Academic

Rescorla RA (1995) Full preservation of a response-outcome association through training with a second outcome. Q J Exp Psychol 48:252-261.
Rescorla RA (1996a) Spontaneous recovery after training with multiple outcomes. Animal Learn Behav 24:11-18. CrossRef

Rescorla RA (1996b) Response-outcome associations remain functional through interference treatments. Animal Learn Behav 24:450-458. CrossRef

Rudebeck PH, Saunders RC, Prescott AT, Chau LS, Murray EA (2013) Prefrontal mechanisms of behavioural flexibility, emotion regulation and value updating. Nat Neurosci 16:1140-1145. CrossRef Medline

Sah P, Faber ES, Lopez De Armentia M, Power J (2003) The amygdaloid complex: anatomy and physiology. Physiol Rev 83:803-834. Medline

Schilman EA, Uylings HB, Galis-de Graaf Y, Joel D, Groenewegen H) (2008) The orbital cortex in rats topographically projects to central parts of the caudate-putamen complex. Neurosci Lett 432:40-45. CrossRef Medline

Schoenbaum G, Takahashi Y, Liu TL, McDannald MA (2011) Does the orbitofrontal cortex signal reward? Ann N Y Acad Sci 1239:87-99. CrossRef Medline

Shiflett MW, Balleine BW (2010) At the limbic-motor interface: disconnection of basolateral amygdala from nucleus accumbens core and shell reveals dissociable components of incentive motivation. Eur J Neurosci 32:1735-1743. CrossRef Medline

Voorn P, Vanderschuren LJ, Groenewegen HJ, Robbins TW, Pennartz CM (2004) Putting a spin on the dorsal-ventral divide of the striatum. Trends Neurosci 27:468-474. CrossRef Medline

Yin HH, Ostlund SB, Knowlton BJ, Balleine BW (2005) The role of the dorsomedial striatum in instrumental conditioning. Eur J Neurosci 22: 513-523. CrossRef Medline 